Check for updates

Cite this: RSC Adv., 2021, 11, 3134

Received 11th December 2020 Accepted 7th January 2021

DOI: 10.1039/dOra10436a

rsc.li/rsc-advances

\section{A more sustainable isothiocyanate synthesis by amine catalyzed sulfurization of isocyanides with elemental sulfur $\dagger$}

\begin{abstract}
R. Nickisch, (1D ${ }^{a}$ P. Conen, ${ }^{a}$ S. M. Gabrielsen (iD ${ }^{a}$ and M. A. R. Meier (iD *ab
Isothiocyanates (ITCS) are typically prepared using amines and highly toxic reagents such as thiophosgene, its derivatives, or $\mathrm{CS}_{2}$. In this work, an investigation of a multicomponent reaction (MCR) using isocyanides, elemental sulfur and amines revealed that isocyanides can be converted to isothiocyanates using sulfur and catalytic amounts of amine bases, especially DBU (down to 2 mol\%). This new catalytic reaction was optimized in terms of sustainability, especially considering benign solvents such as Cyrene ${ }^{\mathrm{TM}}$ or $\gamma^{-}$ butyrolactone $(\mathrm{GBL})$ under moderate heating $\left(40{ }^{\circ} \mathrm{C}\right)$. Purification by column chromatography was further optimized to generate less waste by maintaining high purity of the product. Thus, $E$-factors as low as 0.989 were achieved and the versatility of this straightforward procedure was shown by converting 20 different isocyanides under catalytic conditions, while obtaining moderate to high yields (34-95\%).
\end{abstract}

\section{Introduction}

Isothiocyanates (ITCs) are widely used in heterocycle ${ }^{1-10}$ and thiourea synthesis ${ }^{11-13}$ as well as for medicinal and biochemistry applications (e.g. in the Edman degradation ${ }^{14,15}$ for amino acid sequencing of peptides or as electrophiles in bioconjugates). ${ }^{16-23}$ Several representatives of this group are known to exhibit biological activity, ${ }^{24}$ e.g. anti-cancer, ${ }^{25-33}$ anti-inflammatory, ${ }^{34-38}$ antimicrobial, ${ }^{39}$ antibiotic, ${ }^{39}$ antibacterial, ${ }^{40}$ fungicidal ${ }^{41}$ or insecticidal activity. ${ }^{42,43}$ Commonly, they are synthesized by using thionyl transfer agents (thiophosgene, its derivatives, ${ }^{2,44-48}$ or, more recently, fluorinated agents) ${ }^{49-54}$ or via the formation of dithiocarbamate salts with carbon disulfide under basic conditions, followed by desulfurization (Scheme 1a) ${ }^{44,55-57}$ These approaches are usually chosen due to the easy access of the respective amines, which can be transferred quickly to the ITC in one or two steps. However, these reagents are highly toxic (thiophosgene and carbon disulfide, the latter additionally being volatile) and their synthesis typically involves noxious compounds (i.e. considering the synthesis of thiophosgene, perchloromethyl mercaptan,${ }^{58}$ chlorine and carbon disulfide are used). ${ }^{59}$ Moreover, ITCs are in general noxious compounds (GHS05 and GHS08) and must be handled with care.

${ }^{a}$ Institute of Organic Chemistry (IOC), Karlsruhe Institute of Technology (KIT), Straße am Forum 7, 76131 Karlsruhe, Germany. E-mail: m.a.r.meier@kit.edu; Web: http:// www.meier-michael.com

${ }^{b}$ Institute of Biological and Chemical Systems-Functional Molecular Systems (IBCSFMS), Karlsruhe Institute of Technology (KIT), Straße am Forum 7, 76131 Karlsruhe, Germany

$\dagger$ Electronic supplementary information (ESI) available. See DOI: 10.1039/d0ra10436a
As chemists, we should avoid such problematic functional groups and their synthesis whenever possible, but we cannot stop using these important functional groups completely. Thus, applying in situ approaches to minimize their risks is a typical

\section{a) conventional route}

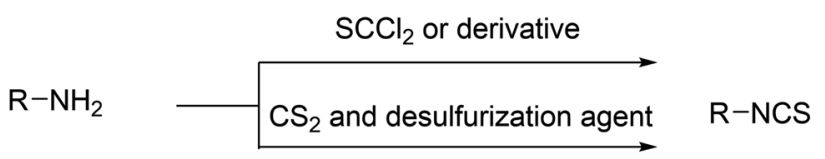

b) alternative route using isocyanide

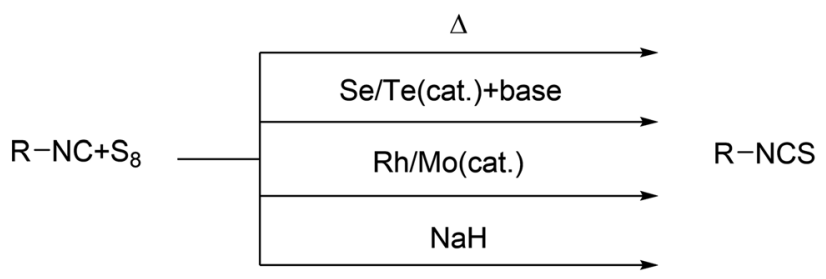

c) this work

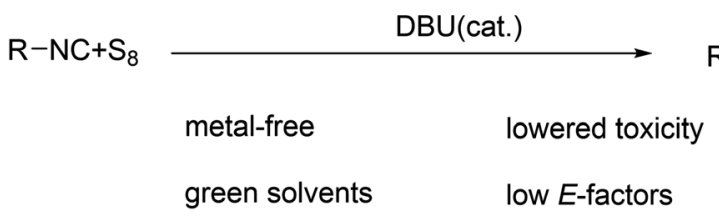

Scheme 1 Overview of reported synthesis protocols towards isothiocyanates. 

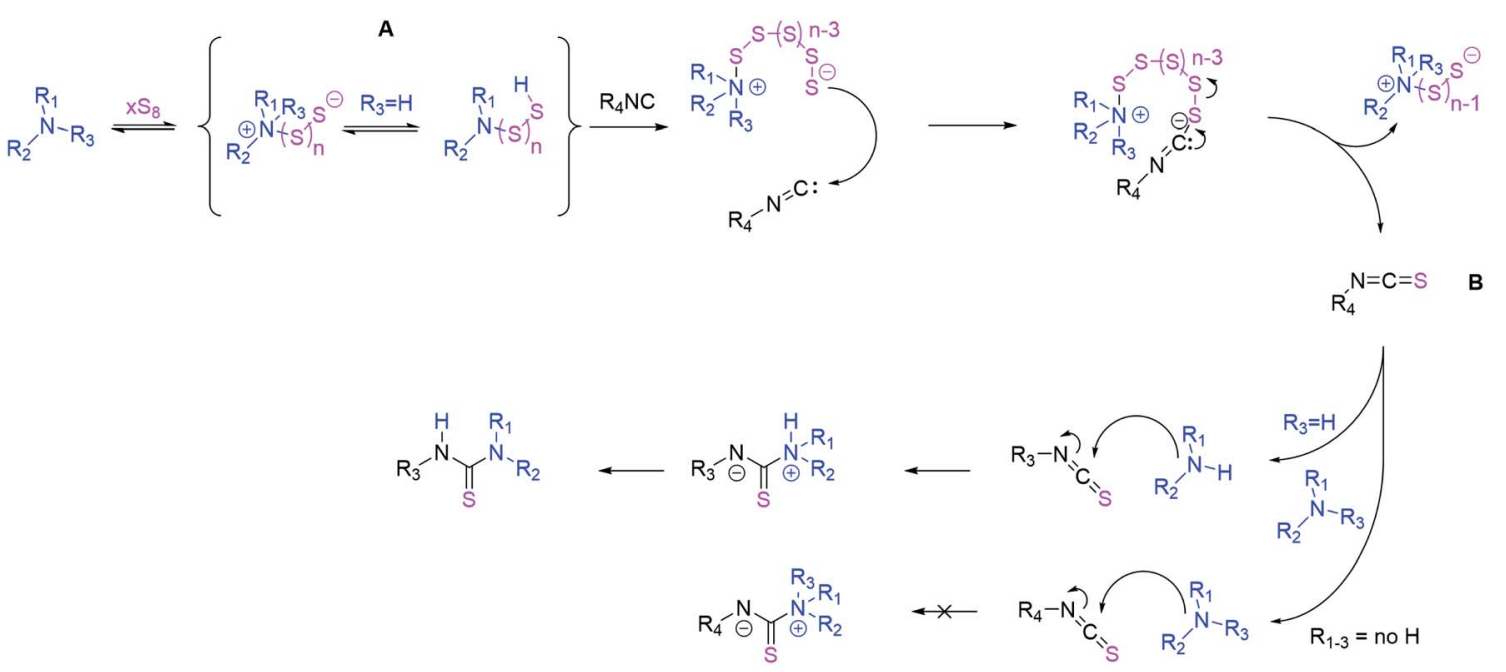

Scheme 2 Possible mechanistic pathway of the MCR between isocyanide (black), elemental sulfur (purple) and an amine (blue), whereby the amine acts as reactant as well as catalyst to form polysulfur chains A. Applying primary or secondary amines leads to thioureas, while tertiary amines lead to isothiocyanates B.

strategy, but only applicable if such compounds can be used as intermediates. Here, we focused our work on a more sustainable synthesis route toward ITCs by considerably reducing the toxicological impact of the used synthesis reagents as well as minimizing the amount of waste ( $E$-factor), two very important aspects in terms of overall sustainability. The above described inherent toxicity of the ITC functional group does of course remain unchanged and thus, these compounds have to be handled with caution. Besides the aforementioned synthesis starting from amines, several other functional groups ${ }^{2,44,45,60-62}$ can be converted to ITCs, of which isocyanides, commonly used in multicomponent reactions (MCRs), ${ }^{63-65}$ are particularly interesting as they are generally less toxic. ${ }^{63}$ The sulfurization of isocyanides with elemental sulfur was previously reported in literature for a few compounds, ${ }^{66,67}$ however this reaction was reported to proceed only at high temperatures $\left(80^{\circ} \mathrm{C}\right.$ and 130 $\left.{ }^{\circ} \mathrm{C}\right)^{66,67}$ and thus, several catalysts have since been reported to allow milder reaction conditions (Scheme 1b). Most commonly, selenium $^{68-70}$ (or tellurium) $)^{71,72}$ in the presence of an amine base efficiently catalyzes the formation of ITCs even at room temperature. Employing several metal catalysts (i.e. molybdenum and rhodium based) led to excellent yields as well. ${ }^{73,74}$ However, selenium and tellurium are highly toxic, and thus we sought to investigate the use of sulfur as suitable sulfurization agent and simultaneously avoiding the use of toxic metal catalyst in this approach. The use of elemental sulfur is highly desired considering the current storage issues of millions of tons of sulfur produced as waste product from the petroleum industry (almost 80 million metric tons in 2019) ${ }^{75}$ due to safety reasons $^{76}$ as well as acidification of soil and water. ${ }^{77}$ Furthermore of importance for the herein described new approach, we recently reported a more sustainable synthesis of non-sterically demanding aliphatic isocyanides,$^{78}$ avoiding toxic compounds such as phosgene, its derivatives or $\mathrm{POCl}_{3}$. This reaction relies on $p$-toluenesulfonyl chloride, which is less toxic than otherwise required reagents and a waste product of the industrial saccharin synthesis. ${ }^{79,80}$ With this in hand, we had all tools we need to pursue the synthesis of ITCs via isocyanides in a more sustainable fashion (Scheme 1c).

In 2019, Ábrányi-Balogh and co-workers reported on a mechanistic investigation of a MCR between isocyanides, elemental sulfur and alcohols or thiols under basic conditions (sodium hydride, 2.00 eq.), obtaining (di)thio carbamates. They showed that ITCs are intermediates in this reaction, which could be isolated in excellent yields for one compound $(2,6-$ dimethylphenyl isothiocyanate). ${ }^{81}$ In an earlier work from $\mathrm{Al}-$ Mourabit and co-workers, a similar MCR was reported ${ }^{82}$ using primary or secondary amines instead of alcohols, leading to thioureas.

This strategy was later on applied for the synthesis of organocatalysts. ${ }^{83}$ In this previous work, ITCs were not confirmed as intermediates, but hypothesized as one possibility of three postulated pathways. In this reaction, no external base was required, as an excess of amine (1.20 eq.) was suggested to catalyze the reaction sufficiently. The then postulated mechanistic pathway, involving the ITC intermediate for this MCR (Scheme 2), entails the nucleophilic attack of an amine to the sulfur ring, thus forming polysulfur chains, which then attack the isocyanide to yield an ITCs after eliminating a $S_{x-1}$ sulfur chain. Since primary or secondary amines were used, the possibly present ITC intermediate was immediately converted to the respective thiourea. Thus, we hypothesized that the use of tertiary amines would prevent further reaction of the ITC and thus allow a catalytic synthesis of ITCs.

We thus started investigating the use of tertiary amines as organocatalysts for the sulfurization of isocyanides to yield ITCs in a more sustainable fashion. We further optimized the reaction conditions (temperature, solvent, stoichiometry) as well as the purification (normally flash column chromatography) to minimize the resulting $E$-factor by maintaining high purity. 


\section{Results and discussion}

\section{Mechanistic investigation of sulfur activation}

We initially evaluated the reactivity of various amines in the reaction of a model isocyanide (n-dodecyl isocyanide 1a) with elemental sulfur by reacting them in stoichiometric amounts in a suspension of DMSO, while monitoring the formation of the respective ITC 3a (Table 1, entries 1-6). In every case, the instant formation of the polysulfur chains was visible, since the reaction mixture turned dark brown (same color change was observed in literature $)^{\mathbf{8 4 , 8 5}}$ as soon as the base was added. As the reaction proceeded, the consumption of the suspended sulfur was also visually observed. GC monitoring of the test reactions revealed that equimolar amounts 1,8-diazabicyclo[5.4.0]undec7-en (DBU) or 1,5,7-triazabicyclo[4.4.0]dec-5-en (TBD) led to quantitative conversion after 2 hours of reaction time (Table 1 , entries 3 and 6, respectively). Nucleophilic aromatic amines, like $N, N$-dimethylamino pyridine (DMAP) and $N$-methyl imidazole (NMI), were less efficient, both resulting in $38 \%$ of conversion, which was attributed to their lower basicity. In general, steric hindrance was deemed less important for the formation of the polysulfur chains, since the conversion of isocyanide 1a observed using the sterically hindered triethylamine (TEA) and 1,4-diazabicyclo[2.2.2] octane (DABCO) were also high, $76 \%$ and $84 \%$, respectively.

Taking into account the basicity of the evaluated amines (Table 1, entries 1-6), it was noted that the most basic amine (TBD) led to the highest conversion, which is consistent with previous reports in which elemental sulfur was activated by strong bases like $\mathrm{NaH}^{81}$ or $\mathrm{K}_{2} \mathrm{CO}_{3} .{ }^{85}$ However, DABCO, exhibiting the second lowest $\mathrm{p} K_{\mathrm{a}}$-value, also showed good performance. Assuming both tertiary amine groups of DABCO are able to activate sulfur in this reaction, the amount of active groups present is doubled compared to the other bases, which might lead to higher conversions in this case. These results show that not only basicity defines the reactivity of the applied amine base, albeit a certain degree of basicity is certainly crucial to activate elemental sulfur.

In a second step, we sought to apply the two best performing amines, i.e. DBU and TBD, in a sub-stoichiometric/catalytic amount, as no side products were detected, while monitoring the reaction with GC (Table 1, entries 7-10). As a highly remarkable result, $10 \mathrm{~mol} \%$ of DBU or TBD were able to convert isocyanide 1a after 22 hours at room temperature with high conversion ( $67 \%$ or $69 \%$, respectively) and the corresponding ITC 3a was obtained. DBU showed better results, i.e. 57\% conversion after only two hours, compared to $36 \%$ conversion for TBD after the same time.

\section{Optimization of the reaction conditions}

Having identified two amine bases (TBD and DBU) that can be applied in substoichiometric amounts, we optimized the reaction conditions addressing as many of the Twelve Principles of Green Chemistry ${ }^{90}$ as possible and further minimized the $E$ factor $^{91}$ (not yet taking the purification steps into account). Initially, when screening the reaction temperature, higher conversions were obtained at higher temperatures, but in the context of sustainability a less energy-consuming procedure is desired and we thus considered moderate temperatures $\left(40^{\circ} \mathrm{C}\right)$ for further experiments. Next, we altered the solvents focusing on greener alternatives for commonly used solvents according to solvent selection guides ${ }^{\mathbf{9 2 - 9 4}}$ (for the complete screening, please see the ESI $\dagger$ ). A clear trend was observed, i.e. polar aprotic solvents like DMSO, GBL and Cyrene ${ }^{\mathrm{TM}}$ (dihydrolevoglucosenone) favored product formation, probably due to their ability to dissolve and stabilize polysulfur chains.

Table 1 GC-screening of various amine bases (bold) for the activation of elemental sulfur, leading to the formation of $n$-dodecylisothiocyanate $3 a$

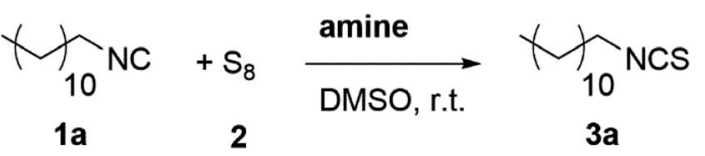

\begin{tabular}{|c|c|c|c|c|}
\hline Entry & Amine $\left(\mathrm{p} K_{\mathrm{a}}\right.$-value in $\left.\mathrm{H}_{2} \mathrm{O}\right)$ & Eq. of amine base & Reaction time/h & Conversion $^{a} / \%$ \\
\hline 1 & DMAP $(9.2)^{86}$ & 1.00 & 2 & 38 \\
\hline 2 & NMI $(7.1)^{87}$ & 1.00 & 2 & 38 \\
\hline 3 & DBU $(11.5)^{86}$ & 1.00 & 2 & 99 \\
\hline 4 & DABCO $(8.9)^{86}$ & 1.00 & 2 & 84 \\
\hline 5 & TEA $(10.7)^{88}$ & 1.00 & 2 & 76 \\
\hline 6 & TBD $(14.5)^{89}$ & 1.00 & 2 & 100 \\
\hline 7 & DBU & 0.10 & 2 & 57 \\
\hline 8 & DBU & 0.10 & 22 & 67 \\
\hline 9 & TBD & 0.10 & 2 & 36 \\
\hline 10 & TBD & 0.10 & 22 & 69 \\
\hline
\end{tabular}

${ }^{a} 1 \mathrm{mmol}$ of $n$-dodecyl isocyanide $1 \mathrm{a}$ was reacted with elemental sulfur ( $2.00 \mathrm{eq}$. of sulfur atoms) and the respective amount of amine in $1 \mathrm{~mL}$ of DMSO at room temperature (r.t.). GC samples were taken after the respective time and conversions were calculated using biphenyl ( 0.25 eq.) as internal standard (IS). 
Table 2 Optimization of reaction conditions (concentration) for the sulfurization of isocyanide 1a via GC-screening to decrease the $E$ factor

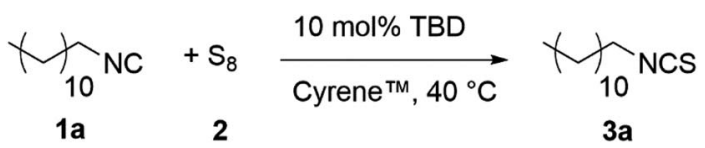

\begin{tabular}{lllcc}
\hline Entry & Solvent & Concentration $^{a} / \mathrm{M}$ & Conversion $^{b} / \%$ & $E_{\text {-Factor }}{ }^{c}$ \\
\hline 1 & - & - & 6.7 & 16.9 \\
2 & Cyrene $^{\mathrm{TM}}$ & 1.0 & 85 & 5.71 \\
3 & Cyrene $^{\mathrm{TM}}$ & 2.0 & 99 & 2.95 \\
3 & Cyrene $^{\mathrm{TM}}$ & 4.0 & 100 & 1.58 \\
4 & Cyrene $^{\mathrm{TM}}$ & 6.0 & 100 & 1.12
\end{tabular}

${ }^{a}$ Concentration of isocyanide $1 \mathrm{a}$ in the solvent. ${ }^{b} 1.00 \mathrm{mmol}$ of $n$ dodecylisocyanide 1 was reacted with elemental sulfur $(2.00$ eq. of sulfur atoms $)$ and $\mathrm{TBD}(10 \mathrm{~mol} \%)$ in the Cyrene $\mathrm{TM}^{\mathrm{TM}}$ at $40{ }^{\circ} \mathrm{C}$. GCsamples were taken after 30 minutes of reaction time and conversions were calculated using biphenyl (0.25 eq.) as IS. ${ }^{c} E$-Factor was calculated assuming conversion equals the yield, as no side-reaction were observed, not taking the purification steps into account.

Amongst them, Cyrene ${ }^{\mathrm{TM}}$ allowed full conversion of isocyanide 1a after two hours reaction time, while GBL and DMSO also led to high conversions (92 and 85\%, respectively). Both GBL and Cyrene $^{\mathrm{TM}}$ are greener alternatives if compared to DMSO and are synthesized from renewable resources (butane-1,4-diol ${ }^{95}$ and cellulose, ${ }^{96}$ respectively), thus decreasing the overall environmental impact. However, more volatile compounds would allow an easier removal and recycling of the solvent. Among the more volatile and sustainable solvents, only acetone was able to achieve good, yet lower conversions (69\%). The contribution of the solvent for the proceeding of this reaction was further underpinned by testing the reaction in absence of any solvent, resulting in a low conversion of $\sim 8 \%$ after 30 minutes (Table 2 , entry 1).
To decrease both reaction time and waste, we increased the concentration of isocyanide 1a up to $6 \mathrm{M}$ in Cyrene ${ }^{\mathrm{TM}}$ (Table 2, entries 2-4), which was the upper limit due to practical reasons (since elemental sulfur was only partially dissolved at the beginning of the reaction, a certain amount of solvent was needed to facilitate stirring). Thus, the $E$-factor could be reduced to 1.12 maintaining full conversion after 30 minutes (Table 2, entry 4). Furthermore, we decreased the excess of elemental sulfur to 1.12 eq. of sulfur atoms, while maintaining high conversion (98\% after 30 minutes). Since $10 \mathrm{~mol} \%$ of catalyst performed excellent under the optimized conditions, we then evaluated lower catalyst loadings of DBU (Table 3), since (i) higher conversions in less amount of time were obtained in prior tests and (ii) the stirring of the reaction is facilitated by DBU being a liquid.

We note however that the toxicity of DBU is higher compared to TBD (DBU is labeled with GHS05, while TBD is labelled as GHS07). As a result of the high concentration of isocyanide 1a in the solvent ( $6 \mathrm{M})$, the catalyst loading of DBU could be further reduced to $1 \mathrm{~mol} \%$ while still achieving nearly quantitative conversion after prolonged reaction times of 20 hours. Using $2 \mathrm{~mol} \%$ of DBU at the same concentration of $1 \mathrm{a}$ resulted in complete conversion after only four hours. For more diluted reaction mixtures $(c$ isocyanide $)=2 \mathrm{M})$, the amount of DBU had to be increased to $5 \mathrm{~mol} \%$ to obtain full conversion after four hours, which is still a low catalyst-loading. The final optimized reaction conditions are depicted in Scheme 3. We note that when a liquid isocyanide was used, the conditions were $2 \mathrm{~mol} \%$ of DBU in $6 \mathrm{M}$ Cyrene ${ }^{\mathrm{TM}}$ solution, while for solid isocyanides $5 \mathrm{~mol} \%$ of DBU in $2 \mathrm{M}$ Cyrene $^{\mathrm{TM}}$ was used, predominantly to facilitate stirring.

\section{Evaluating the scope of the sulfurization of isocyanides}

Having the optimized reaction conditions in hand, we synthesized several mono- and diisothiocyanates to evaluate the substrate scope of our new procedure (Scheme 3). Aliphatic,

Table 3 Optimization of reaction conditions (catalyst loading of DBU, bold) for the sulfurization of isocyanide 1a via GC-screening

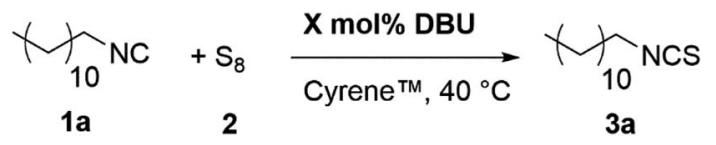

\begin{tabular}{|c|c|c|c|c|}
\hline Entry & Catalysts loading/mol\% & Concentration $^{a} / \mathrm{M}$ & Reaction time/h & Conversion $^{b} / \%$ \\
\hline 1 & 5 & 2.0 & 0.5 & 59 \\
\hline 2 & 5 & 2.0 & 4 & 100 \\
\hline 3 & 2 & 2.0 & 0.5 & 29 \\
\hline 4 & 2 & 2.0 & 20 & 99 \\
\hline 5 & 2 & 6.0 & 4 & 100 \\
\hline 6 & 1 & 6.0 & 4 & 78 \\
\hline 7 & 1 & 6.0 & 20 & 96 \\
\hline
\end{tabular}

${ }^{a}$ Concentration of isocyanide 1a in Cyrene ${ }^{\mathrm{TM}} .{ }^{b} 1.00 \mathrm{mmol}$ of $n$-dodecylisocyanide 1 was reacted with elemental sulfur (1.12 eq. of sulfur atoms) and DBU (respective amount) in Cyrene ${ }^{\mathrm{TM}}$ at $40{ }^{\circ} \mathrm{C}$. GC-samples were taken after the respective time and conversions were calculated using biphenyl (0.25 eq.) as IS. 


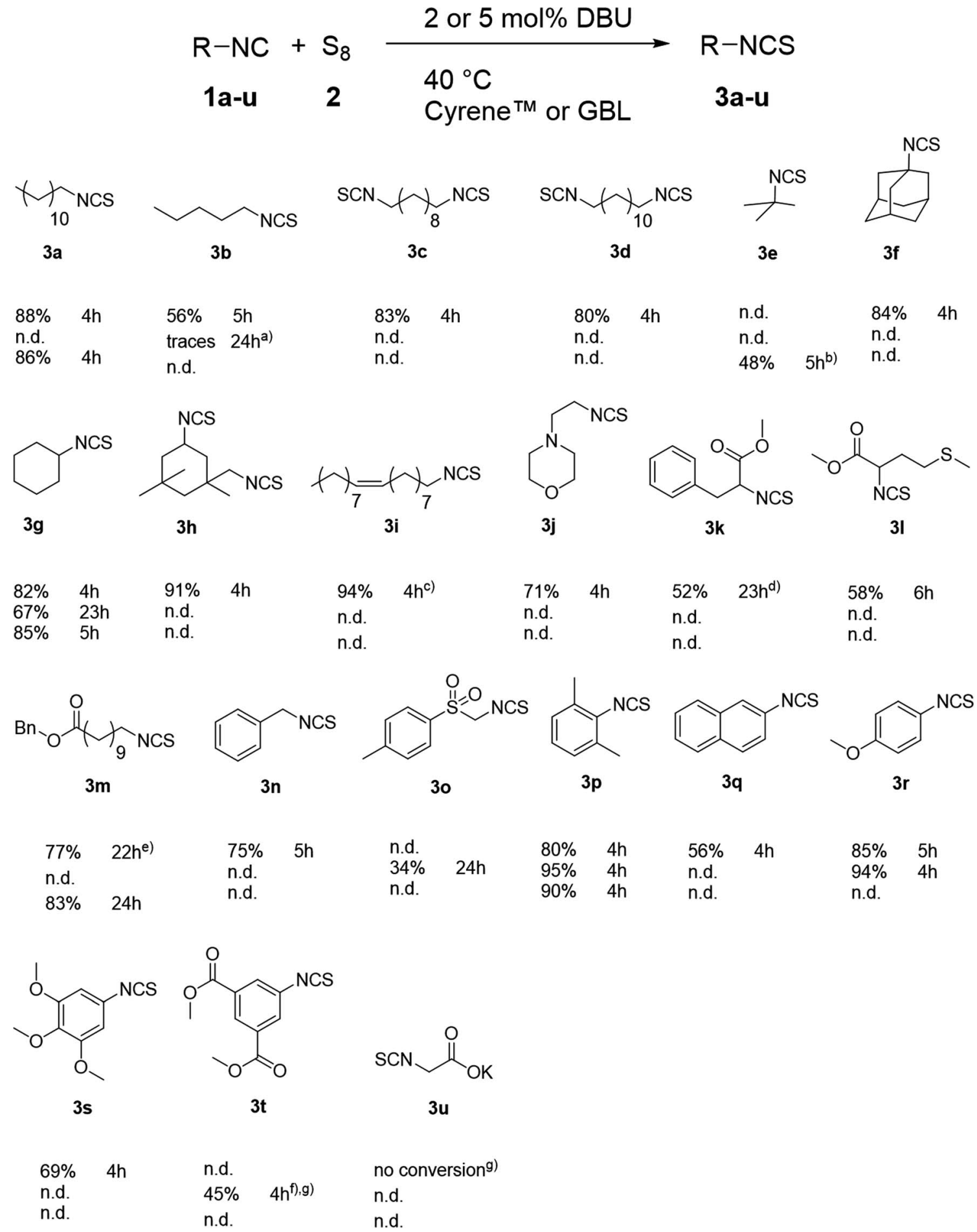

Scheme 3 Herein synthesized ITCs 3a-3t using the new procedure in a $2.5 \mathrm{mmol}$ scale using elemental sulfur ( 1.12 eq. Of sulfur atoms). If the isocyanide was a liquid at $40{ }^{\circ} \mathrm{C}, 2 \mathrm{~mol} \% \mathrm{DBU}$ and $417 \mu \mathrm{L}$ solvent was used, if it was a solid, $5 \mathrm{~mol} \% \mathrm{DBU}$ and $1.25 \mathrm{~mL}$ solvent were used. For each compound, the yield and the reaction time are displayed. The first line corresponds to the reaction using Cyrene ${ }^{\mathrm{TM}}$ as solvent, the second line using GBL. The third line displays the reaction in Cyrene ${ }^{T M}$ in a $15.5 \mathrm{mmol}$ scale. GC-purities were in general >95\% (see ESI $\dagger$ ). (a) GC-purity was not determined (n.d.); (b) pressure vial was used; (c) GC-purity of the starting material $1 \mathrm{i}$ was $80 \%$ and of ITC $3 \mathrm{i} 83 \%$; (d) $2.31 \mathrm{mmol}$ isocyanide $1 \mathrm{k}$ was used; (e) 5 mol\% DBU was used; (f) $2.00 \mathrm{mmol}$ isocyanide 1t was used; (g) starting material was not/less soluble.

benzylic as well as aromatic isocyanides were successfully converted to the respective isothiocyanates. Comparing aliphatic isothiocyanates, steric hindrance was found to have minor impact on the yield, which varied from 34 to $94 \%$. In the case of aromatic isothiocyanates, electron-rich and deficient as well as condensed aromatic isocyanides were successfully converted obtaining yields from 45 to $95 \%$, whereby no trend related to electron density was observed. In addition, the reaction had 


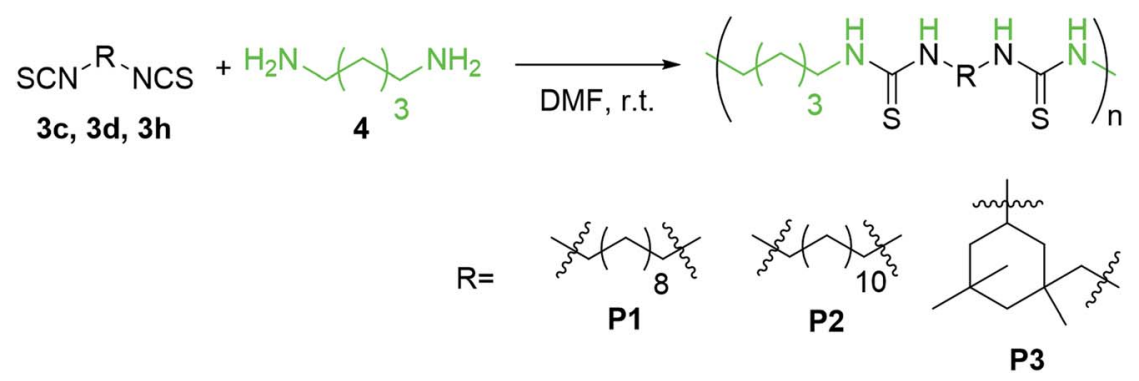

Scheme 4 Synthesis of polythioureas P1-3 with 1,5-diaminopentane 4 (green) and herein synthesized ITCs 3c, 3d and 3h (black).

Table 4 Comparison of sulfurization approaches of isocyanide $3 q$ considering $E$-factors, energy consumption and purification methods

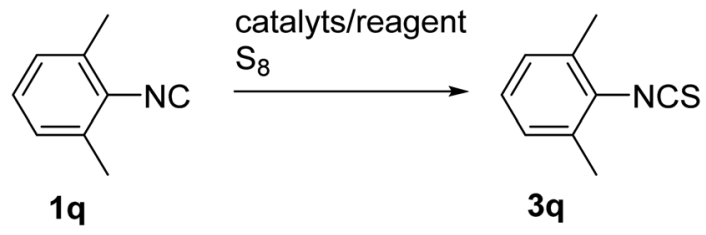

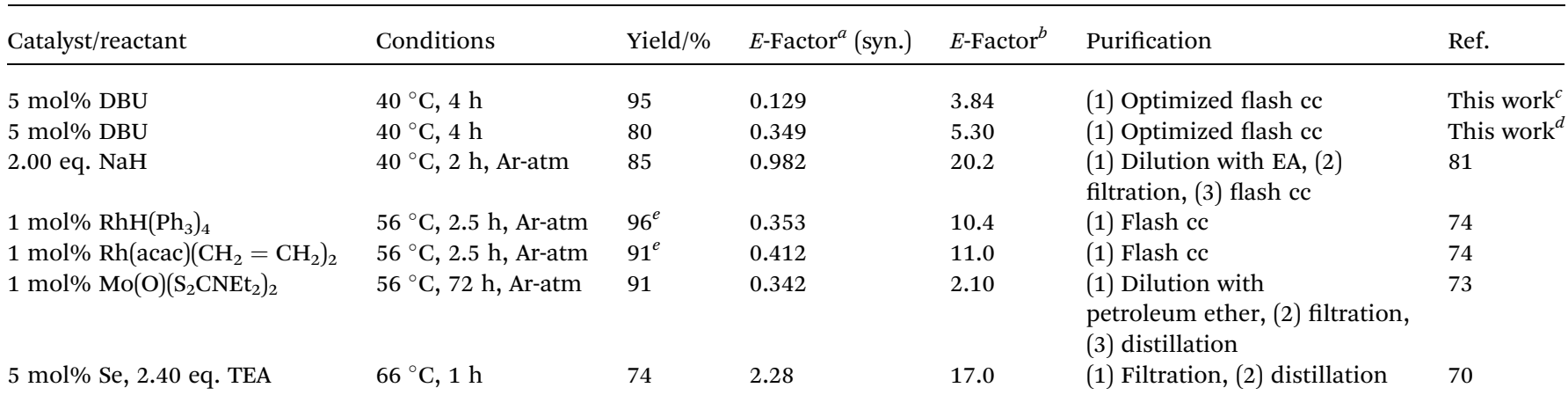

${ }^{a}$ Synthetic $E$-factor involving reactants, catalysts and remaining starting material. ${ }^{b} E$-Factor taking the used reaction solvent into account. ${ }^{c}$ GBL was used as solvent. ${ }^{d}$ Cyrene ${ }^{\mathrm{TM}}$ was used as solvent. ${ }^{e}$ Reference mentioned that high purity of isocyanide was very important. cc $=$ column chromatography.

a high degree of chemoselectivity, since several functional groups (double bond (internal), ether (aliphatic and aromatic), thioether (aliphatic), tertiary amine, $p$-toluenesulfonyl, ester) were tolerated. However, the carboxylic acid salt $3 \mathbf{u}$ (85\% purity) could not be converted, probably due to solubility issues. Considering the tertiary amine of 2-morpholinoethyl isocyanide $3 \mathbf{j}$, an (auto-)catalytic effect might be anticipated, however it was ruled out by performing the reaction in absence of DBU (no yield after 3 hours). Since some isocyanide compounds are volatile (3e), a pressure tube was crucial to obtain the respective product. Besides Cyrene ${ }^{\mathrm{TM}}$, GBL performed very similar as solvent in this reaction, converting isocyanide 1a to ITC 3a and thus, we hypothesized that GBL could be a more suitable solvent for some ITCs. Therefore, we tested six substrates and as a result, better yields were obtained for aromatic ITCs $\mathbf{3 p}$ and $\mathbf{3 r}$, while the yield of ITC $\mathbf{3 g}$ was lower. Synthesis of ITC $\mathbf{3 b}$ in GBL only resulted in minor traces of the product. Nevertheless, in the case of $\mathbf{3 o}$ and $\mathbf{3 t}$, Cyrene ${ }^{\mathrm{TM}}$ exhibited similar retention time on silica gel and thus GBL was found to be more feasible for this reaction in the light of fast and more sustainable purification.
These results showed that GBL can be superior to Cyrene ${ }^{\mathrm{TM}}$ in some cases, depending on the applied substrate.

Purification of all herein reported compounds was performed by a modified flash column chromatography to reduce waste (vide infra). Furthermore, multigram scale reactions (15.5 $\mathrm{mmol}$ ) were easily performed, generally resulting in similar or higher yields (i.e. 3a, 3g, $\mathbf{3 m}$ and $\mathbf{3 p}$ in Scheme 3). This increased yield was likely due to small amounts of sulfur sticking to the wall of the reaction flask, which is a negligible effect for larger scale reactions. All herein newly synthesized compounds were fully characterized by proton and carbon NMR-spectroscopy, IRspectroscopy and high-resolution mass spectrometry. Purities of all synthesized ITCs were determined by gas chromatography and, for several substrates, elemental analysis (vide infra). Furthermore, $E$-factors were calculated for every ITC (see the $\mathrm{ESI} \dagger$ for characterization, purity and $E$-factor data).

\section{Improving sustainability of purification}

Purification of ITCs is commonly performed by (flash) column chromatography, thus adding a considerable amount of waste 
and resulting in poor $E$-factors. Therefore, we sought to optimize the purification procedure as well. Initial attempts to avoid column chromatography by applying several washing steps of the organic layers led to lower purities. Furthermore, excessive washing with aqueous layers was needed and thus, column chromatography was the purification method of choice, resulting in high purity and less waste (GC-purities in general over 95\%, see ESI $\dagger$ ). Ultimately, a dry loaded small column (5$8 \mathrm{~cm}$, see Fig. S1 in ESI $\dagger$ ), similar to a silica plug, was applied as sole purification step directly at the end of the reaction. The amount of used silica, solvent and time was therefore minimized. Residual elemental sulfur was thus also removed, as confirmed by elemental analysis (elemental analysis was performed for several non-volatile ITCs, see ESI $\dagger$ ).

To further underpin the high purity of this less waste producing purification, we performed a polyaddition reaction of diisothiocyanates $\mathbf{3 c}, \mathbf{3 d}$ and $\mathbf{3 h}$ with renewable 1,5-diamino pentane ( $98 \%$ purity) in an equimolar stoichiometry. Since high purities of the monomers, here of the ITCs, are crucial to obtain high molecular weights via step-growth polymerization, a successfully polymerization is a good indication of the suitability of this more sustainable purification (Scheme 4). In all three cases, high molecular weights $\left(M_{\mathrm{n}}\right.$ of P1-3 were $18.6 \mathrm{kDa}$, $55.1 \mathrm{kDa}$ and $58.1 \mathrm{kDa}$, respectively, for SEC-graphs see ESI $\dagger$ ) were obtained, proving that this optimized purification step resulted in excellent purities. However, for compounds $\mathbf{3 o}$ and 3t, applying a classic common column chromatography could not be avoided, since the retention time of the substrate and the solvent were similar to the solvent (vide supra).

\section{Overall sustainability}

Combining the herein reported DBU catalyzed sulfurization of isocyanides with elemental sulfur and the optimized purification step, very low $E$-factors were obtained and the toxicity of the overall reaction was minimized by avoiding highly toxic starting materials. This is underpinned by comparison of the synthesis of compound 3q starting from the respective isocyanide 1q with several literature known procedures (Table 4). For a better comparison of the synthesis protocols, we calculated the $E$ factor with and without the amount of solvent used ( $E$-factor calculated without respect to solvent was herein called synthetic $E$-factor), since some of these procedures would decrease their $E$-factors drastically by conducting the reaction in higher concentrations. Obviously, the use of a catalyzed reaction, compared to reactions using stoichiometric amounts of reagents, resulted in lower $E$-factors. Using the herein reported protocol with Cyrene ${ }^{\mathrm{TM}}$ resulted in the lowest yield of $80 \%$, for which the synthetic $E$-factor was comparable to that of procedures using transition metal catalysts offering higher yields $\left(\mathrm{RhH}\left(\mathrm{Ph}_{3}\right)_{4}\right.$ and $\mathrm{Mo}(\mathrm{O})\left(\mathrm{S}_{2} \mathrm{CNEt}_{2}\right)_{2}$ obtaining 96 and $91 \%$, respectively). On the other hand, the lowest synthetic $E$-factor of 0.129 was obtained using our procedure with GBL as solvent, obtaining an excellent yield as well. Taking the solvent into account, the $E$-factor of our new protocol for the synthesis of $\mathbf{3 q}$ remained very low with 3.84 and 5.30 for GBL and Cyrene ${ }^{\mathrm{TM}}$, respectively. Only the procedure reported by Bargon using a molybdenum catalyst resulted in a lower $E$-factor of 2.10 due to the use of acetone. Nevertheless, even lower $E$-factors, down to 0.989 (for $3 \mathbf{m}$ ), were achieved with our method converting liquid isocyanides (for an overview of all $E$-factors see ESI $\dagger$ ). In addition, this new procedure does not require inert atmosphere, while only moderate energy consumption is needed ( $40{ }^{\circ} \mathrm{C}$ for $\mathrm{ca} .4$ hours). Comparing the purification methods, we minimized the amount of waste produced by purification using an optimized flash column chromatography step as sole purification step. Furthermore, this procedure is metal-free and the overall toxicity of the reaction is minimized by using catalytic amounts of DBU. Nevertheless, the reader has to be reminded that ITCs intrinsically bear a certain degree of toxicity, which must be addressed by applying adequate safety measures.

\section{Conclusion}

Mechanistic investigations of a MCR between isocyanide, amines and elemental sulfur, leading to thioureas, confirmed isothiocyanates as intermediates, which could be trapped herein by using tertiary amines. As a result, a more sustainable synthesis protocol for the sulfurization of isocyanides towards isothiocyanates was established using DBU as greener catalyst in low loading down to $2 \mathrm{~mol} \%$ and the green solvents Cyrene $\mathrm{e}^{\mathrm{TM}}$ and GBL. $E$-Factors down to 0.989 were achieved while toxicity, energy and time consumption of the reaction was minimized obtaining moderate to excellent yields. The sulfurization method tolerates several functional groups and is easily applicable yielding the desired product in high purity. We hope that this procedure can pave the way for greener syntheses of isothiocyanates starting from the respective isocyanides and further the formamides and amines.

\section{Experimental}

\section{General synthesis of isothiocyanates (solid isocyanide)}

The corresponding solid isocyanide (1.00 eq., $\mathrm{mp}>40{ }^{\circ} \mathrm{C}$ ) was dissolved in dihydrolevoglucosenone (Cyrene ${ }^{\mathrm{TM}}$; $c$ (isocyanide) $=2 \mathrm{M}$ ) and elemental sulfur (1.12 eq. of sulfur atoms) was added. After addition of $5 \mathrm{~mol} \%$ of 1,8-diazabicyclo[5.4.0] undec-7-ene (DBU) the reaction mixture was stirred for 4 hours at $40{ }^{\circ} \mathrm{C}$. After completion of the reaction, purification by optimized flash column chromatography applying a dry loaded small column (typically height of silica loading around $5-8 \mathrm{~cm}$, width was $3 \mathrm{~cm}$, see Fig. S1 in ESI $\dagger$ ) and a mixture of cyclohexane and ethyl acetate yielded the pure product.

\section{General synthesis of isothiocyanates (liquid isocyanide)}

The corresponding liquid isocyanide (1.00 eq., $\left.\mathrm{mp} \leq 40{ }^{\circ} \mathrm{C}\right)$ was dissolved in dihydrolevoglucosenone (Cyrene ${ }^{\mathrm{TM}}$; $c$ (isocyanide) $=6 \mathrm{M}$ ) and elemental sulfur (1.12 eq. of sulfur atoms) was added. After addition of $2 \mathrm{~mol} \%$ of 1,8-diazabicyclo[5.4.0] undec-7-ene (DBU), the reaction mixture was stirred for 4 hours at $40{ }^{\circ} \mathrm{C}$. After completion of the reaction, purification by optimized flash column chromatography applying a dry loaded small column (typically height of silica loading around $5-8 \mathrm{~cm}$ 
width was $3 \mathrm{~cm}$, see Fig. S1 in ESI $\dagger$ ) and a mixture of cyclohexane and ethyl acetate yielded the pure product.

\section{Conflicts of interest}

There are no conflicts of interest to declare.

\section{Notes and references}

1 A. K. Mukerjee and R. Ashare, Chem. Rev., 1991, 91, 1-24.

2 S. Sharma, Sulfur Rep., 1989, 8, 327-454.

3 H. Stephensen and F. Zaragoza, J. Org. Chem., 1997, 62, 60966097.

4 J.-W. Qiu, X.-G. Zhang, R.-Y. Tang, P. Zhong and J.-H. Li, Adv. Synth. Catal., 2009, 351, 2319-2323.

5 R. Yella, N. Khatun, S. K. Rout and B. K. Patel, Org. Biomol. Chem., 2011, 9, 3235-3245.

6 S. Guin, S. K. Rout, A. Gogoi, S. Nandi, K. K. Ghara and B. K. Patel, Adv. Synth. Catal., 2012, 354, 2757-2770.

7 J.-J. Chu, B.-L. Hu, Z.-Y. Liao and X.-G. Zhang, J. Org. Chem., 2016, 81, 8647-8652.

8 Y. He, J. Li, S. Luo, J. Huang and Q. Zhu, Chem. Commun., 2016, 52, 8444-8447.

9 S. Khan and C. M. R. Volla, Chem.-Eur. J., 2017, 23, 1246212466.

10 M. Feng, P. Yang, G. Yang, W. Chen and Z. Chai, J. Org. Chem., 2018, 83, 174-184.

11 D. C. Schroeder, Chem. Rev., 1955, 55, 181-228.

12 A. Shakeel, A. A. Altaf, A. M. Qureshi and A. Badshah, J. Drug Des. Med. Chem., 2016, 2, 10-20.

13 F. Steppeler, D. Iwan, E. Wojaczyńska and J. Wojaczyński, Molecules, 2020, 25, 401.

14 P. Edman, Acta Chem. Scand., 1950, 4, 283-293.

15 P. Edman and G. Begg, Eur. J. Biochem., 1967, 1, 80-91.

16 G. Barbarella, Chem.-Eur. J., 2002, 8, 5072-5077.

17 D. Wang, S. Liu, B. J. Trummer, C. Deng and A. Wang, Nat. Biotechnol., 2002, 20, 275-281.

18 S. Dong and M. Roman, J. Am. Chem. Soc., 2007, 129, 1381013811.

19 S. Heckl, A. Sturzu, M. Regenbogen, A. Beck, G. Feil, A. Gharabaghi and H. Echner, Med. Chem., 2008, 4, 348-354.

20 F. Meng, B. N. Manjula, P. K. Smith and S. A. Acharya, Bioconjugate Chem., 2008, 19, 1352-1360.

21 E.-M. Kim, H.-J. Jeong, I.-K. Park, C.-S. Cho, C.-G. Kim and H.-S. Bom, J. Nucl. Med., 2005, 46, 141-145.

22 Y. S. Zhang, R. H. Kolm, B. Mannervik and P. Talalay, Biochem. Biophys. Res. Commun., 1995, 206, 748-755.

23 X. Guo and M. E. Meyerhoff, Appl. Biochem. Biotechnol., 1997, 68, 41-56.

24 K. Srivastava, A. Bhatt, N. Singh, R. Khare, R. Shukla, D. Chaturvedi and R. Kant, Chem. Biol. Interface, 2020, 10, 34-50.

25 E.-S. Hwang and E. H. Jeffery, Food Chem. Toxicol., 2003, 41, 1817-1825.

26 Y. Zhang, L. Tang and V. Gonzalez, Mol. Cancer Ther., 2003, 2, 1045-1052.
27 A. P. Lawson, M. J. C. Long, R. T. Coffey, Y. Qian, E. Weerapana, F. El Oualid and L. Hedstrom, Cancer Res., 2015, 75, 5130-5142.

28 D. Xiao, V. Vogel and S. V. Singh, Mol. Cancer Ther., 2006, 5, 2931-2945.

29 A. Melchini, P. W. Needs, R. F. Mithen and M. H. Traka, J. Med. Chem., 2012, 55, 9682-9692.

30 IARC Handbooks of Cancer Prevention, Cruciferous Vegetables, Isothiocyanates and Indoles, IARC Press, Lyon, 9th edn, 2004.

31 X. Wu, Y. Zhu, H. Yan, B. Liu, Y. Li, Q. Zhou and K. Xu, BMC Cancer, 2010, 10, 269.

32 C. Ioannides and N. Konsue, Drug Metab. Rev., 2015, 47, 356373.

33 M. Traka and R. Mithen, Phytochem. Rev., 2009, 8, 269-282.

34 S. Giacoppo, M. Galuppo, G. R. De Nicola, R. Iori, P. Bramanti and E. Mazzon, Bioorg. Med. Chem., 2015, 23, 80-88.

35 C. Waterman, D. M. Cheng, P. Rojas-Silva, A. Poulev, J. Dreifus, M. A. Lila and I. Raskin, Phytochemistry, 2014, 103, 114-122.

36 M. Galuppo, S. Giacoppo, G. R. De Nicola, R. Iori, M. Navarra, G. E. Lombardo, P. Bramanti and E. Mazzon, Fitoterapia, 2014, 95, 160-174.

37 T. Uto, D.-X. Hou, O. Morinaga and Y. Shoyama, Adv. Pharmacol. Sci., 2012, 2012, 614046.

38 J. V. Cross, J. M. Rady, F. W. Foss Jr, C. E. Lyons, T. L. Macdonald and D. J. Templeton, Biochem. J., 2009, 423, 315-321.

39 D. Li, Y. Shu, P. Li, W. Zhang, H. Ni and Y. Cao, Med. Chem. Res., 2013, 22, 3119-3125.

40 C.-M. Lin, J. F. Preston III and C.-I. Wei, J. Food Prot., 2000, 63, 727-734.

41 H. S. Mayton, C. Olivier, S. F. Vaughn and R. Loria, Phytopathology, 1996, 86, 267-271.

42 V. Borek, L. R. Elberson, J. P. McCaffrey and M. J. Morra, J. Econ. Entomol., 1997, 90, 109-112.

43 L. Williams, M. J. Morra, P. D. Brown and J. P. McCaffrey, J. Chem. Ecol., 1993, 19, 1033-1046.

44 K. Eschliman and S. H. Bossmann, Synthesis, 2019, 51, 17461752.

45 Z. Fu, W. Yuan, N. Chen, Z. Yang and J. Xu, Green Chem., 2018, 20, 4484-4491.

46 S. Kim and K. Y. Yi, J. Org. Chem., 1986, 51, 2613-2615.

47 C. Larsen and D. N. Harpp, J. Org. Chem., 1981, 46, 24652466.

48 C. Larsen, K. Steliou and D. N. Harpp, J. Org. Chem., 1978, 43, 337-339.

49 Y.-Y. Liao, J.-C. Deng, Y.-P. Ke, X.-L. Zhong, L. Xu, R.-Y. Tang and W. Zheng, Chem. Commun., 2017, 53, 6073-6076.

50 W. Feng and X.-G. Zhang, Chem. Commun., 2019, 55, 11441147.

51 J. Yu, J.-H. Lin and J.-C. Xiao, Angew. Chem., Int. Ed., 2017, 56, 16669-16673.

52 T. Scattolin, A. Klein and F. Schoenebeck, Org. Lett., 2017, 19, 1831-1833.

53 J. Wei, S. Liang, L. Jiang and W. Yi, J. Org. Chem., 2020, 85, 12374-12381. 
54 L. Zhen, H. Fan, X. Wang and L. Jiang, Org. Lett., 2019, 21, 2106-2110.

55 S. B. Tsogoeva, D. A. Yalalov, M. J. Hateley, C. Weckbecker and K. Huthmacher, Eur. J. Org. Chem., 2005, 2005, 49955000.

56 P. Molina, M. Alajarin and A. Arques, Synthesis, 1982, 1982, 596-597.

57 Ł. Janczewski, A. Gajda and T. Gajda, Eur. J. Org. Chem., 2019, 2019, 2528-2532.

58 C. H. Hoyt and K. Jonas, Manufacture of perchloromethyl mercaptan, US3014071A, 1961.

59 W. Autenrieth and H. Hefner, Ber. Dtsch. Chem. Ges., 1925, 58, 2151-2156.

60 Z. Shusheng, Z. Tianrong, C. Kun, X. Youfeng and Y. Bo, Eur. J. Med. Chem., 2008, 43, 2778-2783.

61 J. N. Kim, J. H. Song and E. K. Ryu, Synth. Commun., 1994, 24, 1101-1105.

62 M. Baumann and I. R. Baxendale, Beilstein J. Org. Chem., 2013, 9, 1613-1619.

63 A. Dömling and I. Ugi, Angew. Chem., Int. Ed., 2000, 39, 31683210.

64 A. Llevot, A. C. Boukis, S. Oelmann, K. Wetzel and M. A. R. Meier, Top. Curr. Chem., 2017, 375, 66.

65 R. C. Cioc, E. Ruijter and R. V. A. Orru, Green Chem., 2014, 16, 2958-2975.

66 J. H. Boyer and V. T. Ramakrishnan, J. Org. Chem., 1972, 37, 1360.

67 W. Bao, C. Chen, N. Yi, J. Jiang, Z. Zeng, W. Deng, Z. Peng and J. Xiang, Chin. J. Chem., 2017, 35, 1611-1618.

68 K. Nishikawa, T. Umezawa, M. Garson and F. Matsuda, J. Nat. Prod., 2012, 75, 2232-2235.

69 N. A. Weires, E. D. Styduhar, E. L. Baker and N. K. Garg, J. Am. Chem. Soc., 2014, 136, 14710-14713.

70 S. Fujiwara, T. Shink-Ike, N. Sonoda, M. Aoki, K. Okada, N. Miyoshi and N. Kambe, Tetrahedron Lett., 1991, 32, 3503-3506.

71 H. Mitome, N. Shirato, H. Miyaoka, Y. Yamada and R. W. M. van Soest, J. Nat. Prod., 2004, 67, 833-837.

72 S. Fujiwara, T. Shin-Ike, K. Okada, M. Aoki, N. Kambe and N. Sonoda, Tetrahedron Lett., 1992, 33, 7021-7024.

73 W. Adam, R. M. Bargon, S. G. Bosio, W. A. Schenk and D. Stalke, J. Org. Chem., 2002, 67, 7037-7041.

74 M. Arisawa, M. Ashikawa, A. Suwa and M. Yamaguchi, Tetrahedron Lett., 2005, 46, 1727-1729.

75 National Mineral Information Center, Mineral Commodity Summaries, 2020.

76 T. A. Rappold and K. S. Lackner, Energy, 2010, 35, 1368-1380.
77 F. Crescenzi, A. Crisari, E. D'Angel and A. Nardella, Environ. Sci. Technol., 2006, 40, 6782-6786.

78 K. A. Waibel, R. Nickisch, N. Möhl, R. Seim and M. A. R. Meier, Green Chem., 2020, 22, 933-941.

79 C. Fahlberg and I. Remsen, Ber. Dtsch. Chem. Ges., 1879, 12, 469-473.

80 D. J. Ager, D. P. Pantaleone, S. A. Henderson, A. R. Katritzky, I. Prakash and D. E. Walters, Angew. Chem., Int. Ed., 1998, 37, 1802-1817.

81 A. G. Németh, G. M. Keserü and P. Ábrányi-Balogh, Beilstein J. Org. Chem., 2019, 15, 1523-1533.

82 T. B. Nguyen, L. Ermolenko and A. Al-Mourabit, Synthesis, 2014, 46, 3172-3179.

83 R. Nickisch, S. M. Gabrielsen and M. A. R. Meier, ChemistrySelect, 2020, 5, 11915-11920.

84 T. B. Nguyen, M. Q. Tran, L. Ermolenko and A. Al-Mourabit, Org. Lett., 2014, 16, 310-313.

85 W. Cao, F. Dai, R. Hu and B. Z. Tang, J. Am. Chem. Soc., 2020, 142, 978-986.

86 F. Ravalico, S. L. James and J. S. Vyle, Green Chem., 2011, 13, 1778-1783.

87 J. A. Dean, Lange's Handbook of Chemistry, McGraw-Hill, Inc., New York, 15th edn, 1999.

88 I. Kaljurand, A. Kütt, L. Sooväli, T. Rodima, V. Mäemets, I. Leito and I. A. Koppel, J. Org. Chem., 2005, 70, 1019-1028.

89 K. M. K. Yu, I. Curcic, J. Gabriel, H. Morganstewart and S. C. Tsang, J. Phys. Chem. A, 2010, 114, 3863-3872.

90 P. Anastas and J. C. Warner, Green Chemistry: Theory and Practice, Oxford Univeristy Press, Oxford, 1998.

91 R. A. Sheldon, Chem. Ind., 1992, 903-906.

92 K. Alfonsi, J. Colberg, P. J. Dunn, T. Fevig, S. Jennings, T. A. Johnson, H. P. Kleine, C. Knight, M. A. Nagy, D. A. Perry and M. Stefaniak, Green Chem., 2008, 10, 31-36.

93 R. K. Henderson, C. Jimenez-Gonzalez, D. J. C. Constable, S. R. Alston, G. G. A. Inglis, G. Fisher, J. Sherwood, S. P. Binks and A. D. Curzons, Green Chem., 2011, 13, 854862.

94 D. Prat, A. Wells, J. Hayler, H. Sneddon, C. R. McElroy, S. Abou-Shehada and P. J. Dunn, Green Chem., 2016, 18, 288-296.

95 W. Schwarz, J. Schossig, R. Rossbacher, R. Pinkos and H. Höke, in Ullmann's Encyclopedia of Industrial Chemistry, Wiley-VCH, Weinheim, 2019.

96 J. Sherwood, M. De bruyn, A. Constantinou, L. Moity, C. R. McElroy, T. J. Farmer, T. Duncan, W. Raverty, A. J. Hunt and J. H. Clark, Chem. Commun., 2014, 50, 96509652. 